\title{
MS25-03 | How Can We Directly See the Molecule Array in 3D Protein Crystal UNDER TEM?
}

Shintake, Tsumoru (OIST Graduate University, Onna, Okinawa, JPN)

The author discusses on basic physics Bragg diffraction, and propose a new sheme to caputure images under TEM. This technique will directly provide real image of molecules in 3D crystal, and thus suitable to analyze sub-micronsize protein crystals like Micro-ED, even with high mosaic spread, or disordered. The author will also discuss on practical liminations. 